\title{
Localization of Discrete Mobile Scatterers in Vehicular Environments Using Delay Estimates
}

\author{
Martin Schmidhammer, Christian Gentner, Benjamin Siebler \\ Institute of Communications and Navigation \\ German Aerospace Center (DLR) \\ Wessling, Germany \\ Email: \{martin.schmidhammer, christian.gentner, benjamin.siebler\}@dlr.de
}

\begin{abstract}
This paper presents a localization approach which aims to detect and localize discrete mobile scatterers. Therefore, a network of spatially distributed transmitting and receiving nodes is used. The localization problem is formulated as a non-linear optimization problem and corresponding performance bounds for the positioning error are provided. To solve the optimization problem, an iterative non-linear least squares approach is used, following the algorithm of Levenberg and Marquard. The proposed localization approach is evaluated based on wideband measurement data. It is shown, that the localization of mobile scatterers can be achieved. A further evaluation reveals a strong dependence of the localization performance on the preceding link level parameter estimation. Particularly sparse networks are shown to be sensitive to rich multipath environments.

Index Terms-Mulitlateration, localization, parameter estimation, KEST, non-linear least-squares, Levenberg-Marquard
\end{abstract}

\section{INTRODUCTION}

Vehicular environments, in particular in urban space, are typically shared by many different users including cars, motorcycles, cyclists, and pedestrians. With the trend of increasing urbanization, the number of road users steadily grows. The resulting dense and highly complex environment is challenging for any means of automated transport, requiring broad situational awareness to enable safe routing. Therefore, cooperation between road users, i.e. the exchange of user specific information including position and velocity, helps to improve the mutual awareness [1]. Many road users, however, are not equipped with any actively probing devices, i.e. are neither able to determine their own position nor to communicate to others. Hence, realizing reliable situational awareness requires further methods and sensor technologies, which explicitly account for these non-cooperative road users.

Current automated and autonomous vehicles mainly rely on locally mounted sensors to perceive their surroundings, including radar and lidar sensors, as well as camera-based systems. Due to physical properties these locally mounted perception sensors have a series of critical limitations such as their limited performance in occluded sight situations [1]. To improve the reliablity and to extend the awareness range of the ego-perception, supporting infrastructure based systems have been suggested, e.g. based on dedicated radar sensors [2] or cameras [3]. For an appropriate deployment of such systems,

978-1-7281-2445-2/19/\$31.00 C2019 IEEE several sensors would have to be mounted to sufficiently sense only a limited area like an urban intersection, which results in high deployment and maintenance costs.

Therefore, a novel road surveillance system is introduced in [4], proposing to reuse signals from vehicular communications infrastructure for passive radar application. The detection and localization of road users is intended to be achieved by sensing the wireless propagation characteristics between the links of existing communication networks. Thereby, it is assumed that road users and other objects induce delayed and Doppler shifted multipath components (MPC), which correspond to their location and dynamics. Exploiting the network structure and evaluating the individual links allows to infer the location and velocity of road users. A similar idea is followed in [5], where all devices that transmit signals are considered as possible illuminators of opportunity. That means, besides static network nodes also mobile devices can be used. Since the localization accuracy strongly depends on precise location information about the individual network nodes, an incorporation of mobile nodes is challenging and requires to account for location uncertainties.

Following the idea of the surveillance systems of [4] and [5], this paper presents a complementary localization approach. The corresponding measurement model is derived and performance bounds are provided. Eventually, the proposed approach is evaluated based on wideband measurement data.

\section{Network And Measurement Model}

Consider a widely distributed network of $K$ transmitting and $L$ receiving nodes. Both, transmitting and receiving nodes are assumed to be static at known locations $\mathbf{x}_{k}^{\mathrm{Tx}}, k \in\{1, \ldots, K\}$, and $\mathbf{x}_{l}^{\mathrm{Rx}}, l \in\{1, \ldots, L\}$, respectively, where receiving nodes can be collocated with transmitting nodes or individually placed. The transmitting nodes emit known signals $s_{k}(t)$ with period $T_{\mathrm{p}}$ allowing to measure the channel impulse response (CIR) at the receivers [6]. For each link in the network, the received signal is modeled as a superposition of a finite number of scaled and delayed replicas of the transmit signal. These comprise the line-of-sight (LoS) and discrete MPCs due to reflection and scattering. The MPCs are further differentiated according to the dynamics of the scattering objects, i.e. static 
and mobile [7]. Hence, the CIR for a pair of transmitting and receiving nodes $\mathrm{Tx}_{k}$ and $\mathrm{Rx}_{l}$ can be written as

$$
h_{k l}(t, \tau)=h_{k l}^{\mathrm{LoS}}(t, \tau)+h_{k l}^{\mathrm{S}}(t, \tau)+h_{k l}^{\mathrm{M}}(t, \tau),
$$

with $h_{k l}^{\mathrm{LoS}}(t, \tau), h_{k l}^{\mathrm{S}}(t, \tau)$, and $h_{k l}^{\mathrm{M}}(t, \tau)$ denoting the contribution of the LoS, the sum of $P_{k l}$ discrete static and of $Q_{k l}$ discrete mobile MPCs to the CIR, respectively.

Each mobile MPC is attributed to an individual moving scattering object in the observation area. Thereby, the number of mobile MPCs is assumed to be identical for all pairs of transmitters and receivers, i.e. $Q_{k l}=Q$, and uniquely attributable for each link. Eventually, for time and phase synchronized transmitting and receiving nodes, the contribution of discrete mobile scatterers is expressed by

$$
h_{k l}^{\mathrm{M}}(t, \tau)=\sum_{q=1}^{Q} \alpha_{k l q}^{\mathrm{M}}(t) e^{-j 2 \pi f_{\mathrm{c}} \tau_{k l}^{\mathrm{M}}\left(\mathbf{x}_{q}(t)\right)} \delta\left(\tau-\tau_{k l}^{\mathrm{M}}\left(\mathbf{x}_{q}(t)\right)\right)
$$

where $\alpha_{k l q}^{\mathrm{M}}(t)$ represents the complex amplitude and $\tau_{k l}^{\mathrm{M}}\left(\mathbf{x}_{q}(t)\right)$ the propagation delay associated with the $q$ th mobile scatterer at location $\mathbf{x}_{q}(t)=\left[x_{q}(t), y_{q}(t)\right]^{T}$. The term $e^{-j 2 \pi f_{\mathrm{c}} \tau_{k l q}^{\mathrm{M}}\left(\mathbf{x}_{q}(t)\right)}$ denotes the corresponding delay-induced phase shift for center frequency $f_{\mathrm{c}}$. For convenience, in the following the notation for time dependence will be omitted.

The propagation delay is determined by the distances between transmitter and scatterer and between scatterer and receiver as

$$
\begin{aligned}
\tau_{k l}^{\mathrm{M}}\left(\mathbf{x}_{q}\right)=\tau_{k l q}^{\mathrm{M}} & =\frac{1}{c}\left(\left\|\mathbf{x}_{q}-\mathbf{x}_{k}^{\mathrm{Tx}}\right\|+\left\|\mathbf{x}_{q}-\mathbf{x}_{l}^{\mathrm{Rx}}\right\|\right) \\
& =\frac{1}{c}\left(d_{k q}^{\mathrm{Tx}}+d_{l q}^{\mathrm{Rx}}\right)
\end{aligned}
$$

with $c$ as the speed of light, $d_{k q}^{\mathrm{Tx}}$ and $d_{l q}^{\mathrm{Rx}}$ as the distance of scatterer $q$ to $\mathrm{Tx}_{k}$ and $\mathrm{Rx}_{l}$, respectively. The operator $\|\cdot\|$ denotes the standard Euclidean norm. For $K L$ network links, linear independent propagation delays induced by the $q$ th scatterer as defined in (3) compose the vector

$$
\boldsymbol{\tau}_{q}=\left[\tau_{11 q}^{\mathrm{M}}, \ldots, \tau_{K 1 q}^{\mathrm{M}}, \tau_{12 q}^{\mathrm{M}}, \ldots, \tau_{K 2 q}^{\mathrm{M}}, \cdots, \tau_{1 L q}^{\mathrm{M}}, \ldots, \tau_{K L q}^{\mathrm{M}}\right]^{T} .
$$

Given the corresponding vector of measured delays $\hat{\tau}_{q}$, the measurement model is defined as

$$
\hat{\boldsymbol{\tau}}_{q}=\boldsymbol{\tau}_{q}+\mathbf{w}_{q}
$$

with $\mathbf{w}_{q}$ as zero-mean white normal distributed noise with covariance matrix $\boldsymbol{\Sigma}_{q}=\operatorname{diag}\left(\sigma_{k l q}^{2}\right)$.

\section{LOCALIZATION APPROACH}

As stated above, location information of mobile scatterers are contained in the propagation delay of time-variant MPCs. Besides the time-variant MPCs, the CIR between any pair of transmitting and receiving nodes additionally comprise static LoS and MPCs as given in (1). In order to localize mobile scatterers, the time-variant channel components need to be exposed. Therefore, the proposed localization approach is composed of two stages. First, the identification and characterization of LoS and static MPCs in a calibration stage, and second the exposure of mobile MPCs and the actual localization of mobile scatterers in an estimation stage.

For both stages Kalman enhanced super resolution tracking (KEST) is used to estimate and track the channel parameters of the CIR, including complex amplitude and propagation delay of LoS and MPCs [6].

\section{A. Calibration Stage}

To characterize the propagation effects of the static environment, the observation area is assumed to be devoid of any mobile scattering objects, i.e. $Q=0$. For each transmitterreceiver pair, the channel is observed over a period $T_{\text {cal }}$ which results in $\left\lfloor T_{\text {cal }} / T_{\mathrm{g}}\right\rfloor$ consecutive CIR measurements, with $T_{\mathrm{g}}$ as the time interval between two adjacent measurements. Using KEST, the channel parameters for all recorded CIR are estimated. Subsequently, the parameter estimates are clustered with regard to amplitude and delay [8]. The resulting clusters correspond to the LoS and to $P_{k l}$ static MPCs. Each cluster mean and standard deviation are determined as $\bar{\tau}_{k l p}$ and $\bar{\sigma}_{k l p}, p \in\left\{0, \ldots, P_{k l}\right\}$, where $p=0$ denotes the LoS component. Hence, the vectors $\overline{\boldsymbol{\tau}}_{k l}=\left[\bar{\tau}_{k l 0}, \ldots, \bar{\tau}_{k l P_{k l}}\right]^{T}$ and $\overline{\boldsymbol{\sigma}}_{k l}=\left[\bar{\sigma}_{k l 0}, \ldots, \bar{\sigma}_{k l P_{k l}}\right]^{T}$ characterize the static propagation environment between $\mathrm{Tx}_{k}$ and $\mathrm{Rx}_{l}$.

\section{B. Estimation Stage}

Similar to the previous stage, KEST is used to estimate and track the channel parameters of incoming CIRs. As given in (1), the CIR comprises LoS, static and mobile MPCs. Therefore, the vector of propagation delay estimates is composed as

$$
\hat{\boldsymbol{\tau}}_{k l}^{\mathrm{full}}=[\underbrace{\hat{\tau}_{k l 0}^{\mathrm{S}}, \ldots, \hat{\tau}_{k l P_{k l}}^{\mathrm{S}}}_{\text {LoS and static MPC }}, \hat{\tau}_{k l 1}^{\mathrm{M}}, \ldots, \hat{\tau}_{k l Q}^{\mathrm{M}}]^{T} .
$$

In order to expose mobile MPCs, the vector of delay estimates $\hat{\tau}_{k l}^{\text {full }}$ is adjusted with respect to the static components $\overline{\boldsymbol{\tau}}_{k l}$ determined in the preceding calibration stage. Particularly, that means to remove all elements of $\hat{\tau}_{k l}^{\text {full }}$ lying in an interval of $\bar{\tau}_{k l p} \pm 3 \bar{\sigma}_{k l p}$. Here, the 3- $\sigma$ interval ensures to exclude delay estimates assigned as static with a probability higher than $99 \%$. Thereby, it is assumed that the previously determined amount of static MPCs remains constant. The resulting measurement vector of mobile MPCs for the link $\mathrm{Tx}_{k}$ and $\mathrm{Rx}_{l}$ is

$$
\hat{\tau}_{k l}=\left[\hat{\tau}_{k l 1}^{\mathrm{M}}, \ldots, \hat{\tau}_{k l Q}^{\mathrm{M}}\right]^{T} .
$$

Eventually, rearranging the elements of scatterer $q$ over all links in the network gives the measurement vector $\hat{\boldsymbol{\tau}}_{q}$.

On the basis of the measurement model given in (5), the localization of scatterer $q$ can be achieved by maximum likelihood estimation. Using a weighted non-linear least-squares approach [9], [10] to minimize the cost function

$$
\mathbf{L}\left(\mathbf{x}_{q}\right)=\left(\hat{\boldsymbol{\tau}}_{q}-\boldsymbol{\tau}_{q}\right)^{T} \boldsymbol{\Sigma}_{q}^{-1}\left(\hat{\boldsymbol{\tau}}_{q}-\boldsymbol{\tau}_{q}\right)
$$

with respect to the unknown position $\mathbf{x}_{q}$, which yields

$$
\hat{\mathbf{x}}_{q}=\underset{\mathbf{x}_{q}}{\arg \min } \mathbf{L}\left(\mathbf{x}_{q}\right) \text {. }
$$

To solve the non-linear two-dimensional optimization problem of (9) it is necessary to use an iterative approach, as there exists 
no closed-form solution. For minimizing the cost-function (8), the Levenberg-Marquardt algorithm is applied [10], due to high robustness and fast convergence characteristics. Given the elements of the Jacobian matrix $\mathbf{J}\left(\mathbf{x}_{q}\right) \in \mathbb{R}^{K L \times 2}$, i.e. the partial derivatives of $\boldsymbol{\tau}_{q}$ with respect to $\mathbf{x}_{q}$, as

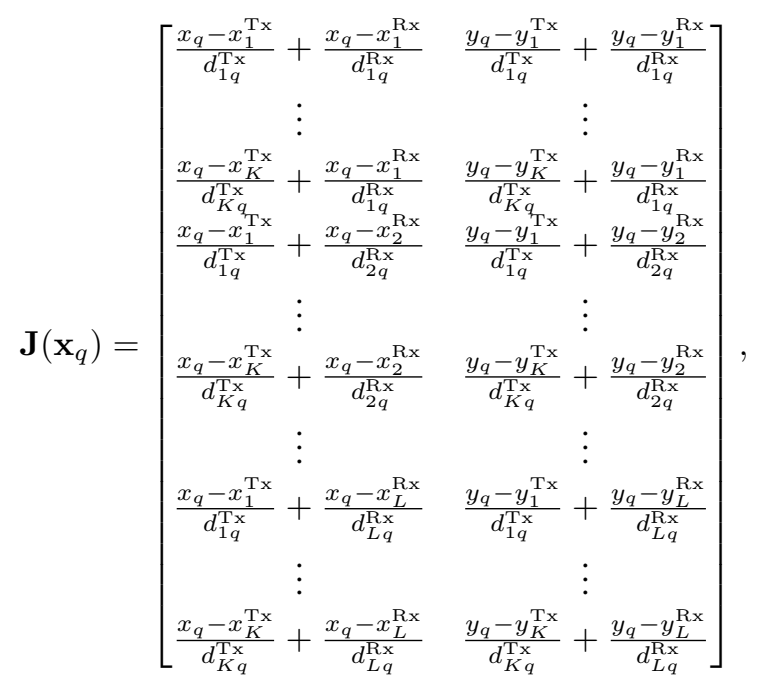

the iterative procedure results in

$$
\begin{array}{r}
\mathbf{x}_{q}^{(i+1)}=\mathbf{x}_{q}^{(i)}+\left(\mathbf{J}^{T}\left(\mathbf{x}_{q}^{(i)}\right) \boldsymbol{\Sigma}_{q}^{-1} \mathbf{J}\left(\mathbf{x}_{q}^{(i)}\right)+\lambda^{(i)} \mathbf{I}\right)^{-1} \\
\mathbf{J}^{T}\left(\mathbf{x}_{q}^{(i)}\right) \boldsymbol{\Sigma}_{q}^{-1}\left(\hat{\boldsymbol{\tau}}_{q}-\boldsymbol{\tau}_{q}^{(i)}\right),
\end{array}
$$

with identity matrix $\mathbf{I}$ and dampening parameter $\lambda^{(i)}$ for iteration step $i$. The individual elements of vector $\boldsymbol{\tau}_{q}^{(i)}$ are calculated according to (3) as $\tau_{k l}^{\mathrm{M}}\left(\mathbf{x}_{q}^{(i)}\right)$.

\section{Performance Bound}

The Cramér-Rao lower bound (CRLB) provides a lower bound on the variance of any unbiased estimator for deterministic parameters. The CRLB is defined as the inverse of the Fisher information matrix (FIM) [9]. Thus, for the vector parameter $\mathbf{x}_{q}$, the elements of the unbiased estimator $\hat{\mathbf{x}}_{q}=\left[\hat{x}_{q}, \hat{y}_{q}\right]^{T}$ satisfy

$$
\operatorname{Var}\left(\hat{x}_{q}\right) \geq\left[\mathbf{F}\left(\mathbf{x}_{q}\right)^{-1}\right]_{1,1}=\sigma_{x_{q}}^{2}
$$

and

$$
\operatorname{Var}\left(\hat{y}_{q}\right) \geq\left[\mathbf{F}\left(\mathbf{x}_{q}\right)^{-1}\right]_{2,2}=\sigma_{y_{q}}^{2}
$$

with $\left[\mathbf{F}\left(\mathbf{x}_{q}\right)^{-1}\right]_{n, n}, n=1,2$, as diagonal elements of the inverse $\operatorname{FIM~} \mathbf{F}\left(\mathbf{x}_{q}\right)$. $\operatorname{Var}(\cdot)$ denotes the variance of an estimator.

For each link in the network, the received signals are a function of propagation delays $\tau_{q}$. Therefore, applying the chain rule to the FIM $\mathbf{F}\left(\mathbf{x}_{q}\right)$ allows an alternative expression as [9]

$$
\mathbf{F}\left(\mathbf{x}_{q}\right)=\mathbf{J}\left(\mathbf{x}_{q}\right)^{T} \mathbf{F}\left(\boldsymbol{\tau}_{q}\right) \mathbf{J}\left(\mathbf{x}_{q}\right)
$$

given the Jacobian matrix $\mathbf{J}\left(\mathbf{x}_{q}\right)$ as defined in (10) and the FIM $\mathbf{F}\left(\boldsymbol{\tau}_{q}\right) \in \mathbb{R}^{K L \times K L}$ with respect to delays $\boldsymbol{\tau}_{q}$. Since the Fisher information for time delay is well known [9], for linear

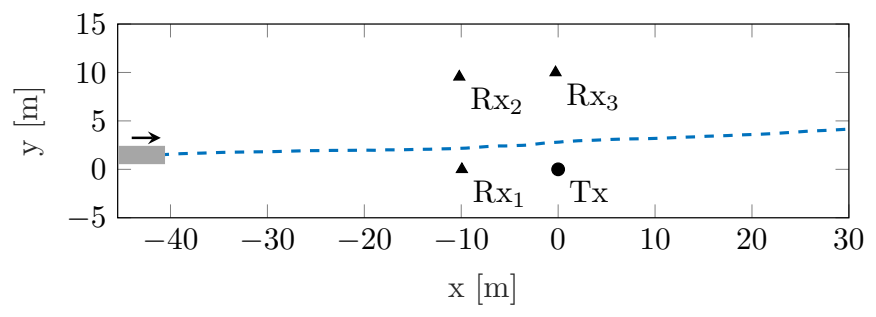

Fig. 1. Overview of the evaluated measurement network with transmitting and receiving nodes as circle and triangles. Moving car and driving direction indicated by grey rectangle and corresponding arrow. Dashed blue line illustrates its trajectory.

independent time delays $\boldsymbol{\tau}_{q}$, the diagonal elements of $\mathbf{F}\left(\boldsymbol{\tau}_{q}\right)$ are defined as

$$
\left[\mathbf{F}\left(\boldsymbol{\tau}_{q}\right)\right]_{m, m}=\frac{8 \pi^{2} \beta^{2} \mathrm{SNR}_{m q}}{c^{2}}
$$

where $\beta^{2}$ denotes the effective bandwidth of the transmit signal. The index $m=1, \ldots, K L$ enumerates vector elements according to the order of network links as introduced in (4). Therewith, $\mathrm{SNR}_{m q}$ expresses the signal-to-noise-ratio (SNR) for the MPC induced by scatterer $q$ in network link $m$. For $\mathrm{Tx}_{k}$ and $\mathrm{Rx}_{l}$ to define link $m$, the $\mathrm{SNR}$ can be written as

$$
\mathrm{SNR}_{m q}=\left(P_{\mathrm{Tx}_{k}} \frac{G_{\mathrm{Tx}_{k}} G_{\mathrm{Rx}_{l}} \sigma_{q} c^{2}}{(4 \pi)^{3} f_{\mathrm{c}}^{2}\left(d_{k q}^{\mathrm{Tx}}\right)^{2}\left(d_{l q}^{\mathrm{Rx}}\right)^{2}}\right) P_{\mathrm{n}}^{-1}
$$

with $P_{\mathrm{Tx}_{k}}$ as transmit power, $P_{\mathrm{n}}$ as receiver noise power, $G_{\mathrm{Tx}_{k}}$ and $G_{\mathrm{Rx}_{l}}$ as antenna gains, and $\sigma_{q}$ as radar cross-section (RCS) of scatterer $q$ [4]. Hence, the FIM elements in (15) are proportional to $\left[\mathbf{F}\left(\boldsymbol{\tau}_{q}\right)\right]_{m, m} \propto\left(\left(d_{k q}^{\mathrm{Tx}}\right)^{2}\left(d_{l q}^{\mathrm{Rx}}\right)^{2}\right)^{-1}$.

Using the CRLB of (12) and (13) allows to analyze the localization accuracy in terms of root mean square error (RMSE), given as

$$
\begin{gathered}
\operatorname{RMSE}_{q}=\sqrt{E\left[\left\|\hat{\mathbf{x}}_{q}-\mathbf{x}_{q}\right\|^{2}\right]} \geq \sqrt{\sigma_{x_{q}}^{2}+\sigma_{y_{q}}^{2}} . \\
\text { V. CASE STUDY }
\end{gathered}
$$

In this section the proposed localization approach is evaluated based on channel measurements for a static network of $K=1$ transmitting and $L=3$ receiving nodes. As shown in Fig. 1, the network nodes are individually placed forming a square with an inter-node distance of approximately $10 \mathrm{~m}$. The selected scenario considers a driving car as single mobile scatterer $(Q=1)$, approaching and passing through the static network setup. As the experiment was conducted outdoors in an open-sky environment, GNSS was used as ground truth system. Therefore, the car was equipped with a Topcon Legacy E+ L1/L2 Glonass/GPS receiver which was connected to a NavExperience $3 \mathrm{G}+\mathrm{C}$ defense antenna. The antenna was placed centrally on the roof of the car. As measurement system the Medav RUSK-DLR wideband channel sounder was used [11]. The measured data are CIRs between transmit and receive antennas, which correspond to network nodes, respectively. Table I provides a summary of the corresponding measurement parameter settings. The individual 
TABLE I

MEASUREMENT PARAMETERS

\begin{tabular}{ll}
\hline Parameter & Value \\
\hline Center frequency $f_{\mathrm{c}}$ & $5.2 \mathrm{GHz}$ \\
Bandwidth $B$ & $120 \mathrm{MHz}$ \\
Signal period $T_{\mathrm{p}}$ & $0.8 \mu \mathrm{s}$ \\
Measurement rate $T_{\mathrm{g}}$ & $1.024 \mathrm{~ms}$ \\
Transmit power $P_{\mathrm{Tx}}$ & $36 \mathrm{dBm}$ \\
Antenna gain $G_{\mathrm{Tx}}$ & $8 \mathrm{dBi}$ (toroidal, omni-directional) \\
Antenna gain $G_{\mathrm{Rx}_{1-3}}$ & $8 \mathrm{dBi}$ (toroidal, omni-directional)
\end{tabular}

static antenna locations of the network are measured prior to the experiment. With the transmit antenna Tx chosen as the origin of the coordinate system, the considered observation area spans from $-45 \mathrm{~m}$ to $30 \mathrm{~m}$ in $x$-direction and from $-5 \mathrm{~m}$ to $15 \mathrm{~m}$ in $y$-direction.

As stated in Sec. III, the channel parameters for all links in the network are estimated using KEST. The estimation results of KEST for the measured CIR over the positions in $x$-direction of the driving car are shown in Fig. 2a-2c for each link individually. The figures show the consecutive vectors of delay estimates (6) over the full traveled distance. Static delay estimates, including LoS and static MPCs, are shown in grey. These static delays together with corresponding standard deviations are determined during a preceding phase without any mobile objects in the observation area applying the steps of the calibration stage (Sec. III-A). This characterization of the static propagation environment allows to expose the mobile MPC, shown in color according to the estimated amplitude level. According to the movement of the car towards the network, passing through it, and moving away from it, the delay estimates of the mobile MPC are characterized by an initially decreasing and later increasing shape. This holds for each link.

Due to the geometrical arrangement of the receiving antennas and limits in the dynamic range of the measurement system, the detection, estimation, and tracking capabilities of KEST differs [11]. Regarding detection range, i.e. the maximum distance which allows to detect the object as an individual MPC, the link for $\mathrm{Tx}-\mathrm{Rx}_{2}$ shows the best performance. This corresponds to the link distance between receive and transmit antenna, which is highest for $\mathrm{Tx}-\mathrm{Rx}_{2}$ among the considered network links. Since the received LoS signal power is lowest, the dynamic range is least stressed. A lower stressed dynamic range, in turn, results in a lower noise floor of the network link. Since scattered MPCs possess very low power, it is obvious that a low noise floor improves the detection and therewith the estimation quality of the corresponding parameters.

Besides detection range, the estimation results of Fig. 2, clearly show how the presence of LoS and static MPCs impact the composition of measurement vector $\hat{\boldsymbol{\tau}}_{q}$ and therewith the localization of the mobile scatterer. An unambiguous solution

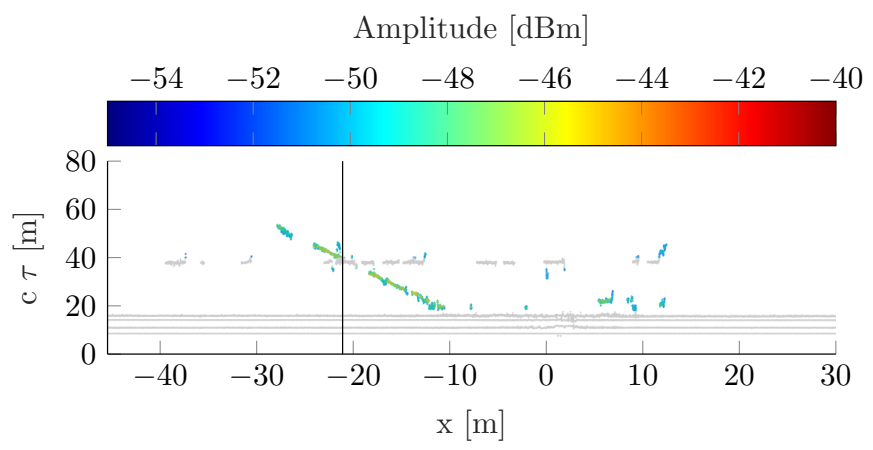

(a) $\mathrm{Tx}-\mathrm{Rx}_{1}$

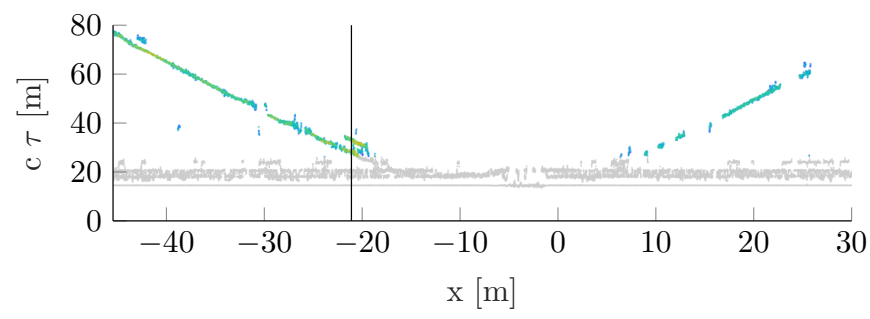

(b) $\mathrm{Tx}-\mathrm{Rx}_{2}$

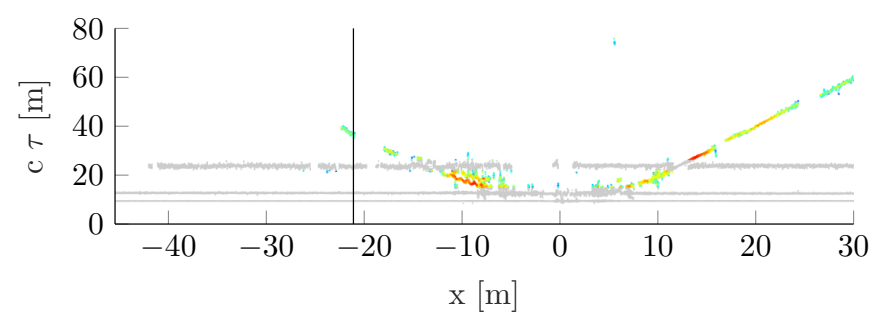

(c) $\mathrm{Tx}_{\mathrm{x}}-\mathrm{Rx}_{3}$

Fig. 2. Estimation results of KEST for the CIR over $x$-positions of the driving car. Exposed mobile MPC is colored according to the estimated amplitude level. LoS and static MPCs are shown in grey. Vertical black lines indicate car position for localization example in Fig. 4.

of (9) requires at least three independent measurements. The required static environment mitigation, i.e. the displacement of any delay estimates close to static components (see Sec. III-B), however, reduces the amount of delay estimates assigned to a specific mobile scatterer. Thus, a rich static MPC environment reduces the overall localization capabilities. This holds particularly for very sparse networks, such as the three-link network considered in this section, since an outage in any link impedes localization. Besides static MPCs, also the LoS impacts the localization capability due to the so called blind zone problem [12]. That means, induced MPCs of scatterers located close to the baseline between a transmitting and receiving node are hardly to detect. The estimation results of the described experiment confirm these blind zones, since it is not possible to expose mobile MPCs when the car is located in the proximity of the individual link baselines. This accounts for the links to $\mathrm{Rx}_{1}$ and to $\mathrm{Rx}_{2}$ in the region between $-10 \mathrm{~m}$ to $0 \mathrm{~m}$, and for the link to $\mathrm{Rx}_{3}$ at around $0 \mathrm{~m}$.

Apart from the link level parameter estimation capabilities, the overall localization performance can be evaluated using 


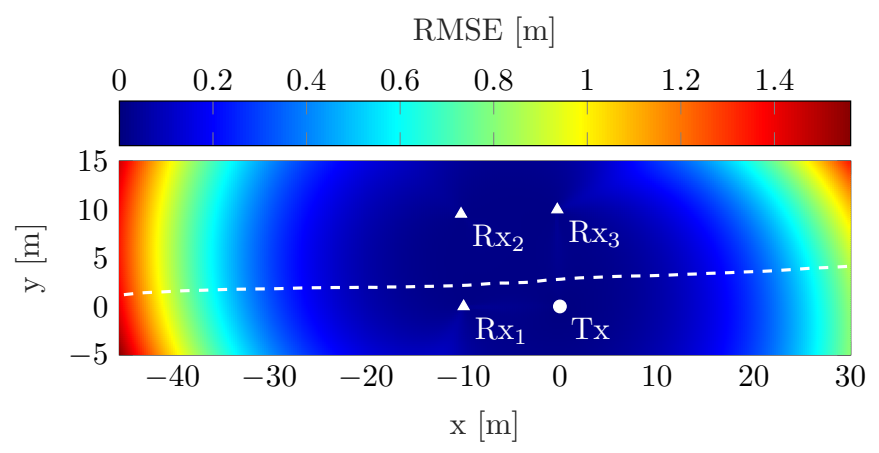

Fig. 3. RMSE for the observation area of the evaluated measurement network Transmitting and receiving nodes as circle and triangles, trajectory of moving car as dashed line.

the RMSE as defined by the CRLB in (17). To calculate the RMSE, the parameters provided in Table I are used and a transmit signal with rectangular power spectral density, i.e. an effective bandwidth of $\beta^{2}=B^{2} / 12$ [9], is assumed. Due to the metallic chassis of the car, a typical value of $100 \mathrm{~m}^{2}$ is used for RCS [4]. Thereby, the RCS accounts for the object's reflectivity characteristics influenced by its size, shape, and material. For the considered observation area, the resulting positioning error is illustrated in Fig. 3. The overall shape of the RMSE indicates good localization performance of scatterers located inside and very close to the network. For distant scatterers the localization performance decreases, which can be explained by both a poor system geometry regarding localization and a lower received power of the echoed signal. Please note, the derived CRLB of Sec. IV only depends on waveform and SNR. That means, the influence of the superposition of LoS and MPCs on the parameter estimation is not considered [13], and would be out of scope of this paper. However, since the superposition of LoS and MPCs strongly impacts the estimation capabilities, e.g. the blind zone problem, its influence on parameter estimation will be included in future research.

Finally, the proposed iterative localization procedure in (11) is applied for 75 consecutive CIR measurements. The time instant of these measurements is indicated by vertical lines in Fig. 2 and the corresponding position of the car is illustrated in Fig. 4a. For the localization approach, corresponding elements of the covariance matrices originating from the delay estimation using KEST are used as measurement noise. The resulting location estimates are shown in Fig. 4. Due to the mobility of the car, the location changes during the considered evaluation interval. Thus, both the ground truth, indicated by the white cross as the center of the car, and the location estimates, indicated by black crosses, are compensated with respect to the absolute movement. Eventually, the estimated location results can be compared to a single ground truth. Thereby, it can be observed that the location estimates differ from the car's center. Accounting for the dimensions of the car, this indicates, that the scattering points are located along the chassis. To analyze the localization accuracy, the CRLB in

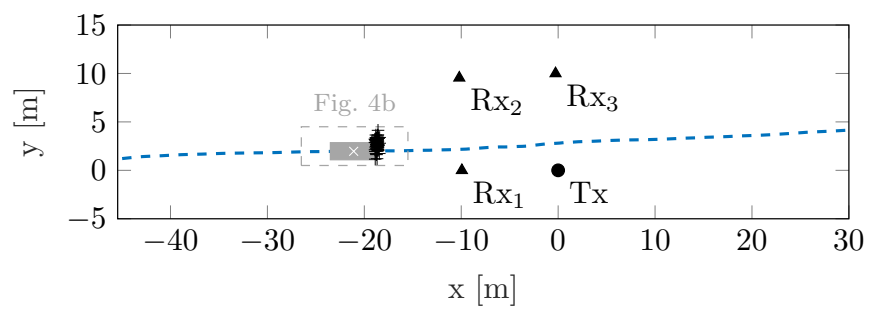

(a) Overview of measurement network and car position for evaluated localization example. Dashed rectangle refers to zoomed illustration in Fig. 4b.

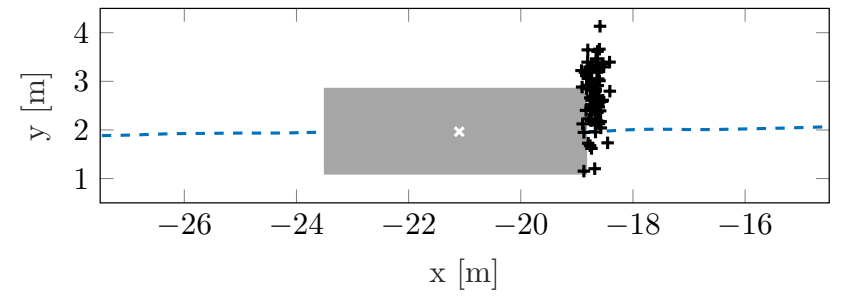

(b) Close up view of car position and location estimates.

Fig. 4. Exemplary location estimates for 75 consecutive CIR. Dimensions of car indicated by grey rectangle. White cross as averaged ground truth located in the center of the car. Black crosses indicate the corresponding location estimates, compensated for mobility. Dashed lines show section of the experiment trajectory.

(12) and (13) are calculated for the selected example position. With a ratio of $\sigma_{y} / \sigma_{x} \approx 5.3$, the theoretical bound of the estimation accuracy in $y$-direction is more than five times higher than in $x$-direction. The distribution of the estimated locations in Fig. 4 can qualitatively confirm this ratio, i.e. the uncertainty in $y$-direction exceeds the uncertainty in $x$ direction. The observed accuracy ratio, however, holds only for the selected example position, since it strongly depends on the system geometry and therewith on the absolute position of the mobile scatterer.

\section{CONCLUSION}

In this paper a localization approach was presented aiming to detect and locate discrete mobile scatterers by means of a network of spatially distributed transmitting and receiving nodes. Therefore, the localization problem was formulated as a non-linear optimization problem. For solving the optimization problem, an iterative non-linear least-squares approach was used, following the algorithm of Levenberg and Marquard. Moreover, the positioning CRLB as performance bound for the stated localization problem was provided.

The proposed localization approach was evaluated based on wideband measurement data. In a case study considering a single mobile scatterer the algorithm's capability to detect and localize a discrete mobile scatterer could be shown. Furthermore, the evaluation revealed challenges in parameter estimation, particularly regarding the mitigation of static LoS and static MPCs in the received signals. Rich multipath environments strongly degrade the availability of usable delay estimates which in turn degrades the localization performance. Thus, an advanced underlying parameter estimation and track- 
ing algorithm, focusing on mobile MPCs, will enhance the localization approach in both performance and robustness.

\section{REFERENCES}

[1] F. de Ponte Müller, "Survey on ranging sensors and cooperative techniques for relative positioning of vehicles," Sensors, vol. 17, no. 2, 2017.

[2] J. M. Munoz-Ferreras, F. Perez-Martinez, J. Calvo-Gallego, A. AsensioLopez, B. P. Dorta-Naranjo, and A. Blanco-del-Campo, "Traffic surveillance system based on a high-resolution radar," IEEE Transactions on Geoscience and Remote Sensing, vol. 46, no. 6, pp. 1624-1633, June 2008.

[3] S. Sivaraman and M. M. Trivedi, "Looking at vehicles on the road: A survey of vision-based vehicle detection, tracking, and behavior analysis," IEEE Transactions on Intelligent Transportation Systems, vol. 14, no. 4, pp. 1773-1795, Dec 2013.

[4] M. Schmidhammer, S. Sand, M. Soliman, and F. de Ponte Müller, "5G Signal Design for Road Surveillance," in 14th Workshop on Positioning, Navigation and Communications (WPNC), October 2017.

[5] R. S. Thomä, C. Andrich, G. D. Galdo, M. Döbereiner, M. A. Hein, M. Käske, G. Schäfer, S. Schieler, C. Schneider, A. Schwind, and P. Wendland, "Cooperative Passive Coherent Location: A Promising Service for Future Mobile Radio Networks," arXiv:1802.04041, March 2018.

[6] T. Jost, W. Wang, U. Fiebig, and F. Perez-Fontan, "Detection and tracking of mobile propagation channel paths," IEEE Transactions on Antennas and Propagation, vol. 60, no. 10, pp. 4875-4883, Oct 2012.
[7] J. Karedal, F. Tufvesson, N. Czink, A. Paier, C. Dumard, T. Zemen, C. F. Mecklenbrauker, and A. F. Molisch, "A geometry-based stochastic MIMO model for vehicle-to-vehicle communications," IEEE Transactions on Wireless Communications, vol. 8, no. 7, pp. 3646-3657, July 2009.

[8] D. Pelleg and A. Moore, "X-means: Extending k-means with efficient estimation of the number of clusters." in Proceedings of the 17th International Conference on Machine Learning (ICML), 2000, pp. 727734.

[9] S. M. Kay, Fundamentals of Statistical Signal Processing: Estimation Theory. Prentice-Hall, 1993.

[10] C. Mensing and S. Plass, "Positioning algorithms for cellular networks using TDOA," in 2006 IEEE International Conference on Acoustics Speech and Signal Processing Proceedings, vol. 4, 2006, pp. 513-516.

[11] M. Schmidhammer, F. de Ponte Müller, S. Sand, and I. Rashdan, "Detection and Localization of Non-Cooperative Road Users based on Propagation Measurements at C-Band," in 12th European Conference on Antennas and Propagation (EuCAP 2018), April 2018, pp. 1-5.

[12] M. Chiani, A. Giorgetti, and E. Paolini, "Sensor radar for object tracking," Proceedings of the IEEE, vol. 106, no. 6, pp. 1022-1041, June 2018.

[13] C. Gentner, T. Jost, W. Wang, S. Zhang, A. Dammann, and U. Fiebig, "Multipath Assisted Positioning with Simultaneous Localization and Mapping," IEEE Transactions on Wireless Communications, vol. 15, no. 9, pp. 6104-6117, Sep. 2016. 